\title{
Perinatal outcomes following maternal asthma and cigarette smoking during pregnancy
}

\author{
Nicolette A. Hodyl' ${ }^{1}$ Michael J. Stark¹, Wendy Scheil'², Luke E. Grzeskowiak and \\ Vicki L. Clifton ${ }^{1}$
}

Affiliations: ${ }^{1}$ Robinson Institute, School of Paediatrics and Reproductive Health, University of Adelaide, Adelaide, and ${ }^{2}$ Epidemiology Unit, Dept of Health, Adelaide, Australia.

Correspondence: V.L. Clifton, Robinson Institute, University of Adelaide, Lyell McEwin Hospital, Haydown Road, Elizabeth Vale, 5112, South Australia, Australia. E-mail: vicki.cliftondadelaide.edu.au

ABSTRACT Does cigarette smoking in pregnancy explain the increased risk of adverse perinatal outcomes that occur with maternal asthma or does it compound the effect?

Using population based birth records, a retrospective analysis was conducted of all singleton pregnancies in South Australia over 10 years $(1999-2008 ; n=172305)$, examining maternal asthma, cigarette smoking and quantity of smoking to estimate odds ratios.

Compared with nonasthmatic females who did not smoke during pregnancy, both asthmatic females who smoked and those who did not smoke during pregnancy had a significantly increased risk of gestational diabetes, antepartum haemorrhage, polyhydramnios, premature rupture of membranes, emergency Caesarean section, and the child being small for gestational age and having congenital abnormalities. These associations suggest that asthma, independently of maternal smoking, increases the risk of these adverse perinatal outcomes. Maternal smoking was itself associated with an increased risk of a number of poor neonatal outcomes, with a dose-response relationship observed. Notably, maternal asthma combined with cigarette smoking significantly increased the risk of preterm birth and urinary tract infections to a greater degree than with either exposure alone.

Maternal asthma and cigarette smoking during pregnancy are both independently associated with adverse perinatal outcomes and, combined, compound the risk of preterm birth and urinary tract infections.

@ERSpublications

Asthma and smoking are independently associated with adverse perinatal outcomes and compound the effect in combination http://ow.ly/s1Bw9

\section{This article has supplementary material available from www.erj.ersjournals.com}

Received: March 272013 | Accepted after revision: July 032013 | First published online: July 302013

Support statement: This work was supported by salary support through National Health and Medical Research Council Fellowships (ID1016379 to N.A. Hodyl, ID565512 to M.J. Stark and ID510703 to V.L. Clifton).

Conflict of interest: None declared.

Copyright @ERS 2014 


\section{Introduction}

Asthma is one of the most common chronic health conditions, with prevalence rates varying between countries from $1 \%$ to $18 \%[1,2]$. Australia has one of the highest rates of asthma internationally, with $12 \%$ of the population affected [3], and particularly high rates in certain subgroups, including females of reproductive age $[4,5]$. Asthma during pregnancy is associated with an increased risk of adverse maternal and neonatal outcomes, including pre-eclampsia, gestational diabetes, perinatal mortality, preterm birth, growth inhibition and congenital malformations [6-11]. These outcomes are most frequently observed with severe asthma, while mild and well-controlled asthma are often associated with no increase in risk [12, 13]. In addition, some studies report differential outcomes with asthma dependent on fetal sex: MURPHY et al. [14] reported female but not male fetal growth inhibition when no inhaled corticosteroid treatment was used during pregnancy, and male but not female growth inhibition in the presence of an asthma exacerbation. Furthermore, CLARK et al. [15] observed an increased frequency of small for gestational age (SGA) male but not female infants born to females with asthma relative to nonasthmatic females. However, others report an absence of sex differences or adverse pregnancy outcomes associated with asthma [16-18].

Active cigarette smoking and exposure to second-hand smoke in pregnancy are independently associated with increased perinatal morbidity and mortality [19-23]. Inconsistent findings relating to the impact of asthma in pregnancy might then be due to an inability to discriminate the effects of asthma from those of cigarette use, with cigarette smoking rates higher in asthmatic than nonasthmatic populations $[3,24]$. Furthermore, previous studies have not adequately investigated whether maternal smoking acts solely as a confounder in this relationship or whether it has an added effect in combination with asthma. In addition to this, the dose-response relationship between asthma and the quantity of cigarettes smoked during pregnancy has been poorly described, despite evidence suggesting quantity of cigarettes is a strong determinant of pregnancy outcomes [25]. Therefore, the aim of this study was to answer the question "does cigarette smoking in pregnancy explain the increased risk of adverse perinatal outcomes that occur with maternal asthma or does it compound the effect?"

\section{Materials and methods}

The South Australian Pregnancy Outcome (Statistics) Unit (Adelaide, Australia) collects information for every live birth and every stillbirth over 20 weeks gestation or $400 \mathrm{~g}$ birth weight on a supplementary birth record form according to the South Australian Health Commission (Pregnancy Outcome Statistics) Regulations 1999. Prior to this information being coded into a database, the information is cross-checked with obstetric health records for accuracy. The information in the perinatal statistics collection has been previously validated and has been shown to be very reliable when compared with hospital case records [26]. We extracted information relating to all births from January 1, 1999 to December 31, 2008 ( $\mathrm{n}=183798$ ). Multiple births were the only exclusion criterion, due to the increased independent risk of adverse outcomes in these pregnancies $(n=5769)$. The study was approved by the human research ethics committees of the South Australian Department of Health and the University of Adelaide (Adelaide, Australia).

Females with asthma were identified using data on maternal illness during pregnancy routinely collected by midwives. The midwives recorded the presence of asthma if females were on medication to prevent or treat asthma or had symptoms of asthma during the current pregnancy. No data are recorded on asthma severity or type of asthma medications used during pregnancy. Females are routinely asked about active smoking of tobacco cigarettes at their first antenatal visit and the number of cigarettes smoked per day after 20 weeks gestation. These data were used to classify females as smokers or non-smokers, and further classify them according to quantity of cigarettes smoked each day in the second half of pregnancy $(0,1-10,11-20$ or $\geqslant 21)$. Females who reported quitting during pregnancy were classified as smokers.

Maternal age was converted into a categorical variable $(<20,20-24,25-29,30-34$ and $>35$ years). Parity was classified as $0,1,2$ or $\geqslant 3$ births. Maternal race was classified as Caucasian, Aboriginal/Torres Strait Islander, Asian or other. Socioeconomic status (SES) for each female was determined using her residential postcode at the time of delivery. Females were then ranked into one of five quintiles according to their level of advantage, or relative disadvantage, based on data from the Socio-Economic Indexes for Areas, calculated from the Australian Bureau of Statistics' 5-yearly census of population and housing [27]. These indices are widely used measures of relative SES.

Maternal medical conditions present in the current pregnancy were recorded on the database from midwives' coding forms. These included six conditions that were identified by ticking a box to indicate their presence in pregnancy. These conditions included asthma, pre-existing diabetes and hypertension, epilepsy, anaemia and urinary tract infection (confirmed by bacteriological culture of urine). Other conditions were recorded according to the International Classification of Diseases (ICD) version 9. Obstetric complications included gestational diabetes, antepartum haemorrhage due to placental abruption, placenta praevia or 
unknown causes. Hypertensive diseases of pregnancy were recorded and included pregnancy-induced hypertension and pre-eclampsia. Additionally, other obstetric complications identified by the midwives were coded in ICD-9, and this study reviewed reports of eclampsia (ICD 6426), polyhydramnios (ICD 6570), placenta praevia without bleeding (ICD 6411/6419) and chorioamnionitis (ICD 6584), which was defined as infection of the amniotic cavity as determined using clinical criteria by the attending physician. Premature labour was regarded as spontaneous onset of labour before 37 weeks gestation, and premature rupture of membranes (PROM) was defined as spontaneous rupture for $>24 \mathrm{~h}$ prior to labour onset. Method of birth included vaginal, assisted (forceps or ventouse), elective caesarean (planned prior to the spontaneous onset of labour) and emergency (undertaken for a complication either before or during labour) Caesarean sections.

The estimated length of gestation at delivery is recorded in the perinatal statistics collection and is based on the last menstrual period and ultrasound examination. Prematurity was defined as birth before 37 weeks completed gestation, with very preterm birth defined as birth before 32 weeks. Birth weight was recorded in grams, and converted to a centile according to an algorithm for Australian births, incorporating gestational age, ethnicity, sex, parity and maternal height (version 6.4; www.gestation.net). SGA was defined as less than the 10th centile, with intrauterine growth restriction (IUGR) defined as less than the third centile. Neonatal resuscitation included intubation or external heart massage at birth. Oxygen therapy for $>4 \mathrm{~h}$ was recorded. Requirement for neonatal or paediatric intensive care with the length of stay in days was recorded. Stillbirth was defined as fetal death at or after 20 weeks gestation. Neonatal death was defined as death within 28 days of birth. A congenital abnormality was defined as any abnormality of prenatal origin, including structural, genetic, chromosomal and biochemical abnormalities, and was categorised according to these criteria or system affected.

Pregnancy outcomes were compared among three different groups in comparison to a control (reference) group. The control group subjects were nonasthmatic females who did not smoke cigarettes during pregnancy, and the three comparator groups were nonasthmatic females who smoked cigarettes during pregnancy, asthmatic females who did not smoke cigarettes during pregnancy and asthmatic females who smoked cigarettes during pregnancy. Outcomes were also analysed according to neonatal sex, given the preexisting evidence for sex-specific differences of asthma on perinatal outcomes [15, 28]. Univariate and multivariate logistic regression modelling were used to identify factors associated with pregnancy and perinatal outcomes, adjusting for potential confounding factors where appropriate, and are reported as odds ratios with $95 \%$ confidence intervals. The presence of effect modification was examined by including an interaction term between asthma and smoking in each logistic regression model, with smoking during pregnancy entered as a dichotomous variable. The correlation matrix between variables in each regression analysis was used to assess collinearity. We included interaction terms between SES and asthma and SES and smoking, and found that these terms did not add significantly to the regression model, and were therefore not included in the final models. Further analysis was performed to assess whether a dose-response relationship was observed between the quantity of cigarettes smoked per day during pregnancy $(0,1-10$, $11-20$ or $\geqslant 21)$ and the risk of poor perinatal outcomes. To avoid amplification of any observed associations due to inclusion of females with previous births during the study period, we repeated each analysis using only primiparous females (as females were not individually identified and, therefore, subsequent pregnancies could not be linked). All analyses were conducted using SPSS, version 17.0 (SPSS, Inc., Chicago, IL, USA) with the significance level set at $\mathrm{p}<0.05$.

\section{Results}

A total of 178029 singleton births were recorded over the 10-year period, with 5724 of these having unknown smoking status during pregnancy. We therefore analysed the outcomes for 172305 births. Asthma was reported in $6.7 \%(n=11512)$ of all births, and a quarter of these pregnancies were associated with maternal smoking, compared with only $17 \%$ in the nonasthmatic population (table 1). Pregnant females who smoked were $\sim 2.5$ years younger than nonsmokers in both the asthmatic and nonasthmatic groups. A higher rate of teenage pregnancy $(<20$ years) and fewer pregnancies in females aged $>35$ years were observed in asthmatic compared with nonasthmatic females in both the smoking and nonsmoking groups. $>90 \%$ of the cohort were Caucasian and were born in Australia. Regardless of asthmatic status, a greater proportion of females who smoked lived in areas characterised by a low socioeconomic index, were more likely to be born in Australia than overseas, and more frequently attended hospitals as public rather than private patients, compared with nonsmokers. The prevalence of smoking during pregnancy decreased over the 10 years, but was consistently higher in the asthma compared with the nonasthmatic group in each year assessed (data not shown).

The frequency of pre-existing medical conditions and pregnancy complications varied according to asthma and smoking status (table 2). Maternal asthma and smoking during pregnancy were both independently 
TABLE 1 Maternal demographics of the study cohort according to maternal asthma and cigarette smoking status during pregnancy

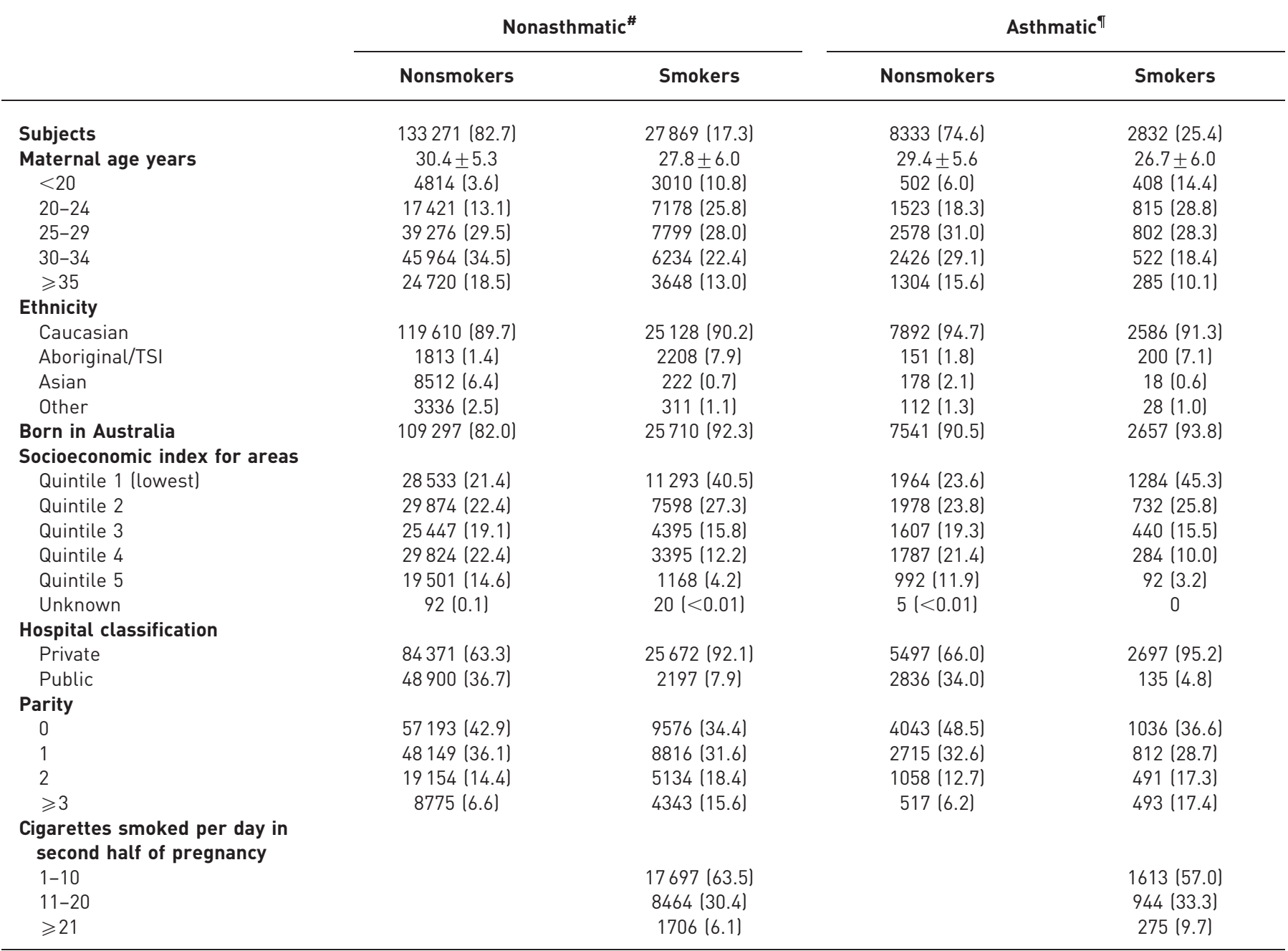

Data are presented as $\mathrm{n}(\%)$ or mean \pm SD. TSI: Torres Strait Islander. ${ }^{*}: \mathrm{n}=161140$ (93.3\%); ${ }^{\circ}: \mathrm{n}=11165$ (6.7\%).

associated with an altered risk of a range of adverse maternal outcomes, with these risks remaining after controlling for a number of potential confounding factors, including maternal age, parity, SES, year of birth, and pre-existing hypertension and diabetes (table 3). Compared with nonasthmatic nonsmokers, both asthmatic smokers and asthmatic nonsmokers had a significantly increased risk for gestational diabetes, antepartum haemorrhage (aetiology unknown), emergency Caesarean sections, polyhydramnios and PROM (table 3). This indicates that asthma contributes to these poor outcomes, independently of maternal smoking. Compared with nonasthmatic nonsmokers, smoking during pregnancy in both the asthmatic and nonasthmatic groups was associated with an increased risk of polyhydramnios and PROM, as well as antepartum haemorrhage (abruption, placenta praevia and that of unknown aetiology), and a significant decrease in the risk for hypertensive disorders of pregnancy and elective Caesareans. A dose-dependent increase in the risks of these adverse outcomes was observed with an increasing number of cigarettes smoked per day, in both the nonasthmatic and asthmatic groups (online supplementary table S1). The combination of maternal asthma and cigarette smoking during pregnancy was associated with a statistically significant increased risk of urinary tract infections compared with either exposure alone, providing evidence of a compound effect. The restriction of the analysis to primiparous females confirmed the increased risk of poor maternal outcomes with either maternal smoking, asthma or both, with few exceptions (online supplementary table S2). Notably, the interaction effect on the risk of urinary tract infections with maternal smoking and asthma was not observed; however, the increased risk of this complication was observed with each individual exposure. 
TABLE 2 Maternal clinical characteristics according to asthma and smoking status during pregnancy

\begin{tabular}{|c|c|c|c|}
\hline & Nonasthmatic & Asthmatic & Total \\
\hline Subjects & 166517 & 11512 & 178029 \\
\hline Pre-existing hypertension & $2022(1.2)$ & 218 (1.9) & 2240 (1.3) \\
\hline Nonsmoker & $1712(1.3)$ & $166(2.0)$ & $1878(1.3)$ \\
\hline Smoker & $310(1.1)$ & $52(1.8)$ & $362(1.1)$ \\
\hline Pre-existing diabetes & $825(0.5)$ & $99(0.9)$ & $924(0.5)$ \\
\hline Nonsmoker & $647(0.5)$ & $60(0.7)$ & 707 (0.5) \\
\hline Smoker & $178(0.6)$ & $39(1.4)$ & $217(0.7)$ \\
\hline Hypertensive disorders of pregnancy & $11958(7.2)$ & $1118(9.7)$ & $13076(7.3)$ \\
\hline Nonsmoker & $10363(7.8)$ & 906 (10.9) & $11269(8.0)$ \\
\hline Smoker & $1595(5.7)$ & $212(7.5)$ & $1807(5.9)$ \\
\hline Gestational diabetes & $6354(3.8)$ & $544(4.7)$ & $6868(3.9)$ \\
\hline Nonsmoker & $5393(4.0)$ & $409(4.9)$ & $5802(4.1)$ \\
\hline Smoker & $961(3.4)$ & $135(4.8)$ & $1096(3.6)$ \\
\hline \multicolumn{4}{|l|}{ Antepartum haemorrhage } \\
\hline Abruption & $1013(0.69)$ & $105(0.9)$ & $1118(0.6)$ \\
\hline Nonsmoker & $731(0.5)$ & $56(0.7)$ & $787(0.6)$ \\
\hline Smoker & $282(1.0)$ & $49(1.7)$ & $331(1.1)$ \\
\hline Placenta praevia & $944(0.6)$ & $75(0.7)$ & $1019(0.6)$ \\
\hline Nonsmoker & $746(0.6)$ & $53(0.6)$ & $799(0.6)$ \\
\hline Smoker & $198(0.7)$ & $22(0.8)$ & $220(0.7)$ \\
\hline Unknown cause & $3814(2.2)$ & $356(3.1)$ & $4170(2.3)$ \\
\hline Nonsmoker & $2927(2.2)$ & 238 (2.9) & $3165(2.2)$ \\
\hline Smoker & $887(3.2)$ & $118(4.2)$ & 1005 (3.3) \\
\hline Urinary tract infection & $3897(2.3)$ & 443 (3.8) & $4340(2.4)$ \\
\hline Nonsmoker & $2794(2.1)$ & 294 (3.5) & $3088(2.2)$ \\
\hline Smoker & $1103(4.0)$ & 149 (5.3) & $1252(4.1)$ \\
\hline Polyhydramnios & $721(0.4)$ & $76(0.7)$ & $797(0.4)$ \\
\hline Nonsmoker & $549(0.4)$ & $51(0.6)$ & $600(0.4)$ \\
\hline Smoker & $172(0.6)$ & $25(0.9)$ & $197(0.6)$ \\
\hline Elective Caesarean & $21099(12.7)$ & $1548(13.4)$ & $22647(12.7)$ \\
\hline Nonsmoker & $18128(15.6)$ & $1213(16.9)$ & $19341(13.7)$ \\
\hline Smoker & $2971(11.7)$ & $335(12.9)$ & $3306(10.8)$ \\
\hline Emergency Caesarean & $25642(15.4)$ & $2056(17.9)$ & $27698(15.6)$ \\
\hline Nonsmoker & $21662(18.7)$ & 1577 (21.9) & $23239(16.4)$ \\
\hline Smoker & 3980 (15.7) & $479(18.5)$ & $4459(14.5)$ \\
\hline Premature labour & $5223(3.1)$ & $428(3.7)$ & $5651(3.2)$ \\
\hline Nonsmoker & $3825(2.9)$ & $249(3.0)$ & $4074(2.9)$ \\
\hline Smoker & $1398(5.0)$ & $179(6.3)$ & $1577(5.1)$ \\
\hline PROM & $3201(1.9)$ & $275(2.4)$ & $3476(2.0)$ \\
\hline Nonsmoker & $2372(1.8)$ & $173(2.1)$ & $2545(1.8)$ \\
\hline Smoker & $829(3.0)$ & $102(3.6)$ & $931(3.0)$ \\
\hline Isoimmunisation & $468(0.3)$ & $30(0.3)$ & $498(0.3)$ \\
\hline Nonsmoker & $364(0.3)$ & $22(0.3)$ & $386(0.3)$ \\
\hline Smoker & $104(0.4)$ & $8(0.3)$ & $112(0.4)$ \\
\hline Last pregnancy stillbirth & $608(0.4)$ & $52(0.5)$ & $660(0.3)$ \\
\hline Nonsmoker & $480(0.5)$ & $34(0.6)$ & $514(0.4)$ \\
\hline Smoker & $128(0.6)$ & $18(0.8)$ & $146(0.5)$ \\
\hline
\end{tabular}

Data are presented as $\mathrm{n}$ or $\mathrm{n}(\%)$. PROM: premature rupture of membranes.

The associations between asthma, smoking and neonatal outcomes

Poor neonatal clinical outcomes were more frequently observed in pregnancies associated with maternal asthma compared with those with no asthma, and in females who smoked compared with nonsmokers (table 4). Maternal asthma and smoking during pregnancy were both independently associated with an increased risk of a range of adverse neonatal outcomes (table 5), with these risks remaining after controlling for a number of potential confounding factors, including maternal age, parity, socioeconomic status, year of birth and pre-existing hypertension and diabetes. In both the asthmatic nonsmoker group and the asthmatic smoker group, an increased risk for SGA and congenital malformations was observed relative to nonasthmatic nonsmokers. This indicates that asthma, independent of maternal smoking, contributes to 


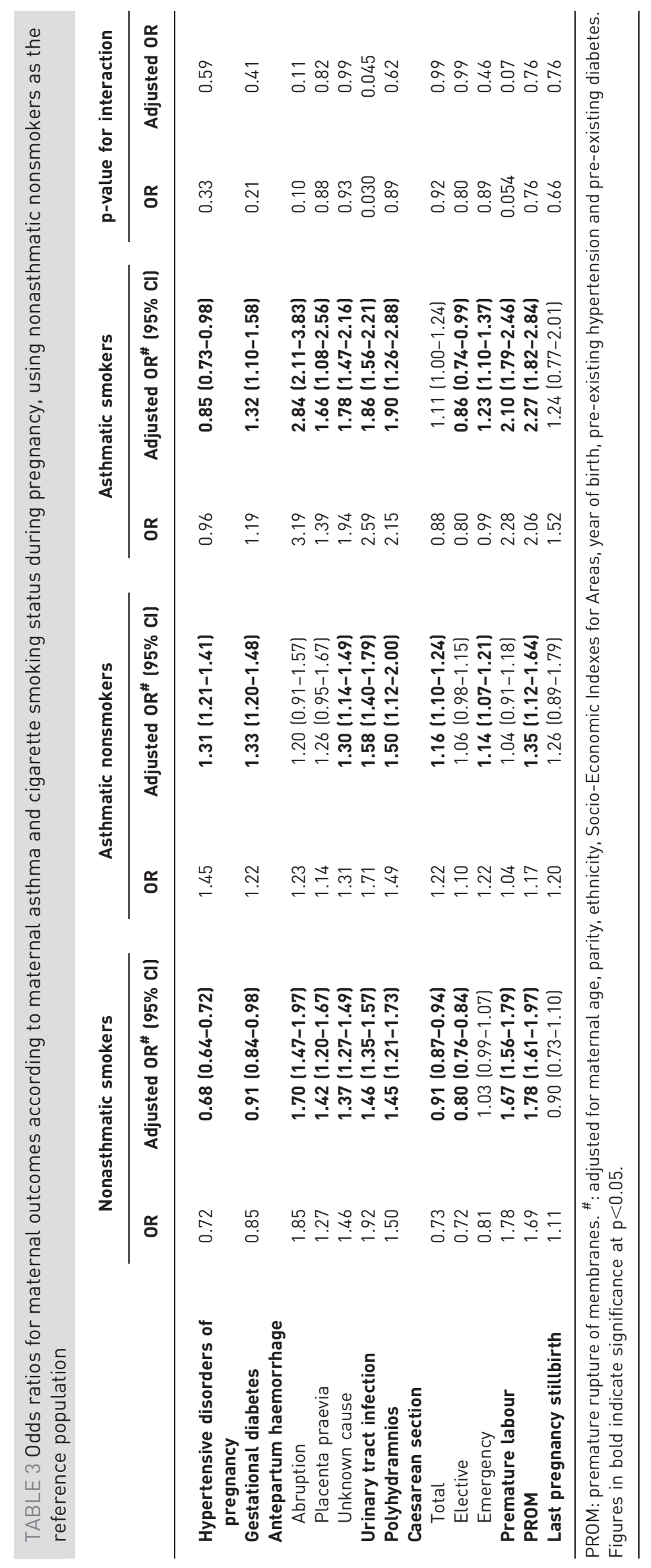


TABLE 4 Neonatal clinical characteristics according to maternal asthma and smoking status during pregnancy

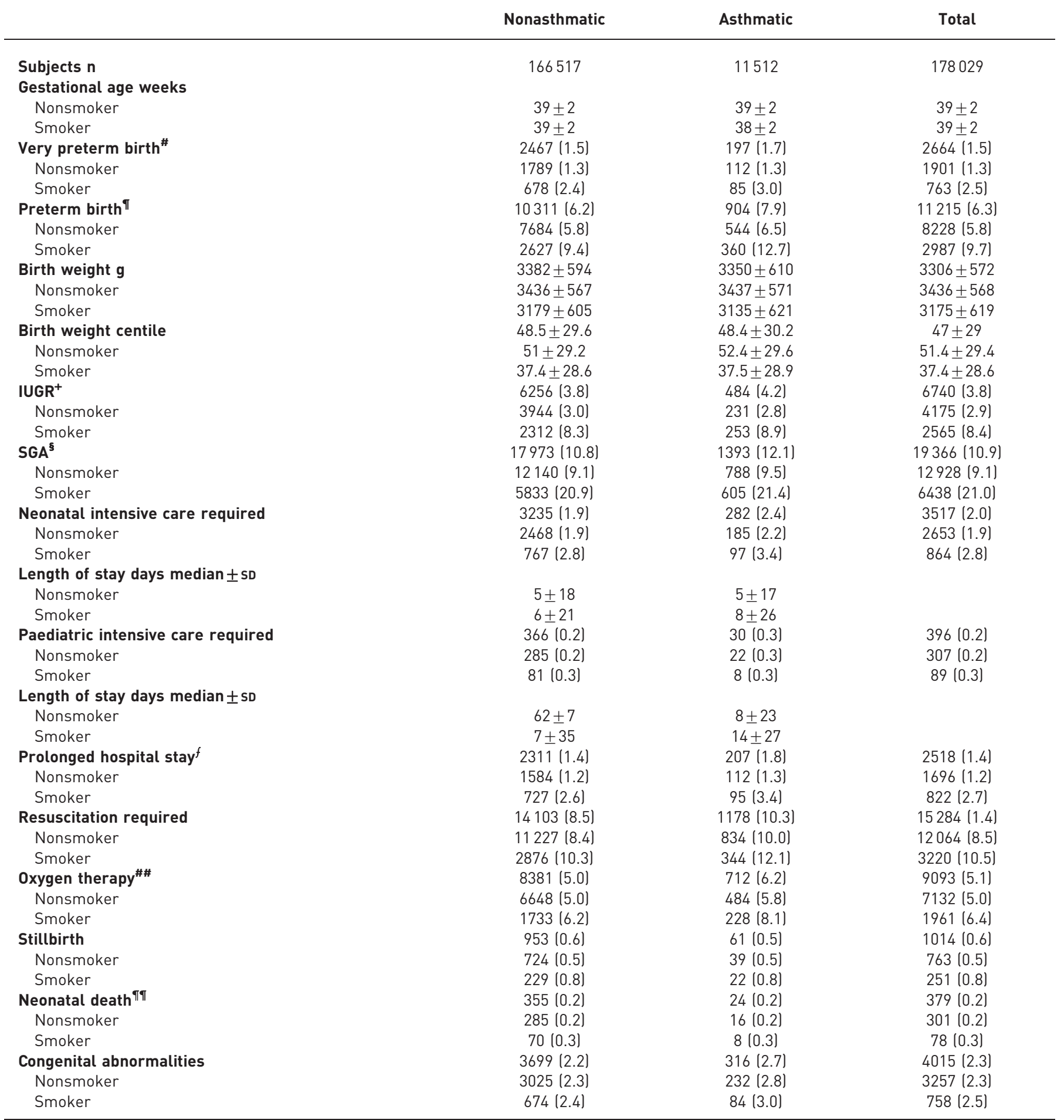

Data are presented as mean \pm SD or $n(\%)$, unless otherwise stated. IUGR: intrauterine growth restriction; SGA: small for gestational age.

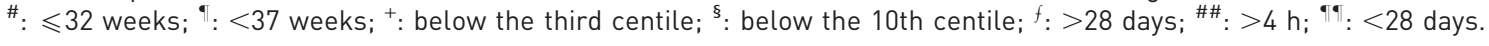

these poor outcomes. Conversely, smoking and not maternal asthma contributed to the increased risk of very preterm birth, IUGR, neonatal intensive care admissions and oxygen therapy following birth, as indicated by the increased odds ratios observed in both nonasthmatic and asthmatic smokers, relative to 


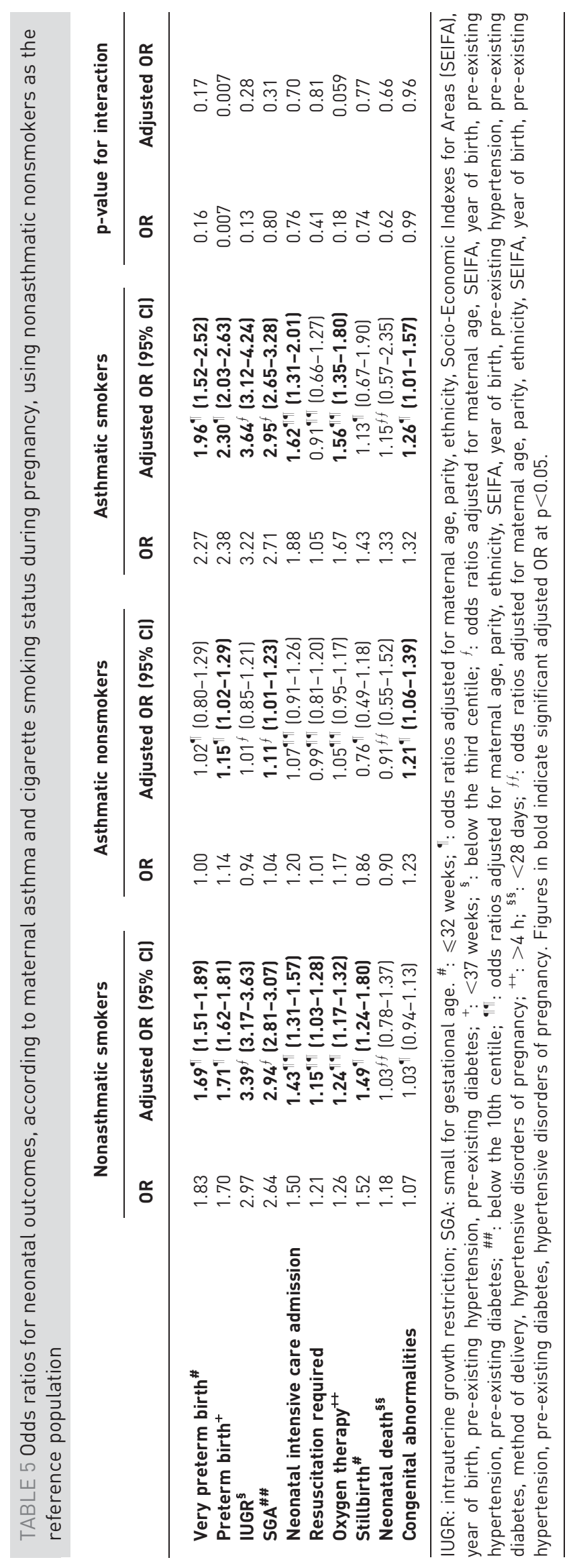




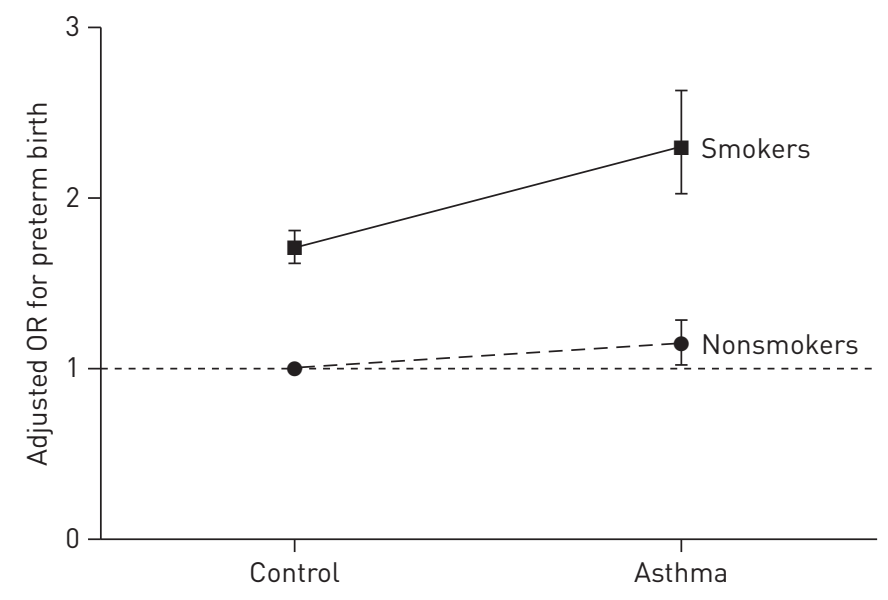

FIGURE 1 The adjusted odds ratios for preterm birth according to maternal asthma and cigarette smoking in pregnancy. The combination of maternal asthma and cigarette smoking during pregnancy was associated with a statistically significant $(p<0.01)$ increased risk of preterm birth than with either exposure alone.

nonasthmatic nonsmokers (table 4). For each of these outcomes, a clear dose-response relationship was observed between the number of cigarettes smoked per day and perinatal outcomes (online supplementary table S1). Notably, the combination of maternal asthma and cigarette smoking was associated with a statistically significant increased risk of preterm birth compared with either exposure alone, providing evidence that maternal asthma and smoking during pregnancy compound to increase the risk for preterm birth (fig. 1). This effect remained when the analysis was restricted to primiparous females (online supplementary table S3).

When the analysis was restricted to primiparous females, the risk of each of the poor neonatal outcomes associated with smoking in the entire cohort remained significant, with additional increased risks observed for neonatal resuscitation and still births (online supplementary table S3). While most adverse neonatal outcomes were observed in both male and female births, an increased risk for SGA deliveries in pregnancies with asthma was observed in pregnancies with a male neonate (adjusted OR (95\% CI) 1.11 (1.03-1.21)), but not with a female, after adjusting for potential confounding variables. No other sex-specific effects were observed.

\section{Discussion}

This is the first epidemiological analysis to qualify the impact of maternal asthma alone and in combination with maternal cigarette use on maternal pregnancy and neonatal outcomes. This 10-year retrospective cohort study assessing perinatal outcomes in the largest Australian population to date confirms that asthma in pregnancy is associated with increased poor outcomes for the mother and neonate. These poor outcomes include hypertensive disorders of pregnancy, gestational diabetes, antepartum haemorrhage (unknown aetiology), Caesarean sections (total and emergency Caesarean sections), premature labour, urinary tract infections, polyhydramnios, PROM, preterm birth, SGA and congenital abnormalities. Furthermore, and importantly, many of these associations were independent of cigarette smoking during pregnancy, while the risk for other adverse outcomes (specifically, urinary tract infections and preterm birth) were further increased in pregnant asthmatic females who smoked cigarettes during pregnancy. Similar to previous reports, we also observed that asthma increased the risk of SGA following birth in male but not female neonates. Overall, these findings suggest that like cigarette smoking during pregnancy, asthma should be recognised and treated as a serious condition that complicates pregnancy for both the mother and her developing infant.

Some debate exists regarding whether the adverse maternal and neonatal outcomes that are associated with asthma are due to the presence of this disease or to underlying common risk factors related to poor health, including cigarette smoking or low SES. In this study, asthma was more prevalent in areas with low compared with high SES, and higher rates of cigarette smoking and pre-existing poor health conditions were observed in the asthmatic compared with the nonasthmatic group. Following multivariate analyses adjusting for the potential impact these factors may have on pregnancy outcomes, asthma was still associated with a significantly increased risk of poor maternal and neonatal outcomes. These results clearly indicate that asthma, independent of maternal smoking during pregnancy, low SES or pre-existing conditions, adversely affects both maternal and neonatal pregnancy outcomes. These data have important clinical implications, suggesting that the appropriate antenatal management (and control) of asthma during pregnancy has the potential to reduce rates of adverse pregnancy outcomes. 
Active cigarette smoking and exposure to second-hand smoke during pregnancy have long been recognised as contributing to poor maternal and neonatal health outcomes [29], as well as longer-term adverse health outcomes for the offspring [30-33]. Adverse perinatal outcomes previously identified include preterm birth, where a dose related impact has been observed [25]. Preterm birth rates are also commonly increased in pregnancies complicated by asthma $[6,7,34]$. The current results suggest that maternal smoking does not act solely as a confounder on preterm birth rates, but may also play an important role in modifying associations between maternal asthma and perinatal outcomes. That is, the combination of maternal smoking and asthma had a multiplicative effect on the risk of preterm birth. Furthermore, the current study demonstrated a dose-response relationship, with increasing number of cigarettes smoked per day in pregnancy increasing the risk of preterm birth in both asthmatic and nonasthmatic females. This may explain the inconsistencies between studies, with some but not all reporting an elevated risk of preterm birth with asthma. Smoking cessation prior to or during pregnancy is recognised as one of the few modifiable risk factors for preterm birth. From these data, we suggest that smoking cessation is particularly important in pregnancies of asthmatic females to decrease the risk of preterm birth. It is evident that there is potential for significant improvements in clinical outcomes to occur with the active inclusion of quit smoking campaigns in the antenatal management of these at-risk pregnancies.

In the current study, the reporting of smoking in pregnancy relied on self-report during an antenatal appointment. This commonly underestimates true smoking prevalence [35], and indeed, the rates in the current study in the nonasthmatic females (17\%) are lower than rates in the adult female Australian population during this time (20\%) [36]. Smoking rates in the asthmatic population were higher than in the nonasthmatic population, a finding consistent with a number of other studies of asthma in pregnancy in different populations $[6,7,24,37]$. Some evidence suggests that smoking may induce asthma via increasing oxidative stress and exacerbating lung inflammation or reducing the efficacy of the bronchial mucosa barrier allowing greater allergen penetrance [38]. Furthermore, smoking reduction in asthmatic patients reduces airway inflammation, improves lung function and improves sensitivity to inhaled corticosteroids [39], supporting an association between smoking and asthma. As both exposures alone and in combination increase the incidence of poor outcomes, and smoking appears to exacerbate asthma, this evidence suggests that females with asthma should be strongly encouraged to quit smoking before or during pregnancy, and reduce exposure to second-hand cigarette smoke to reduce the risk of a poor perinatal outcome.

The current study reported an increased risk of hypertensive disorders of pregnancy with maternal asthma in pregnancy but a decreased risk in females who smoked, both in the asthmatic and nonasthmatic groups. A reduction in hypertensive disorders is commonly observed with cigarette smoking in pregnancy: a metaanalysis of 48 studies identified a reduction of up to $50 \%$ in pre-eclampsia when mothers smoked cigarettes during pregnancy [40]. The mechanism leading to this outcome is currently unknown, but may include changes to maternal and placental angiogenic factors, endothelial function and immune responses, induced by cigarette smoking, all of which contribute to this disease pathology. In support of this, a recent study found an association between smoking in pregnancy and reduced levels of the angiogenic factor soluble FMS-like tyrosine kinase-1 in maternal circulation [41]. Further research is required to elucidate the organs effecting and affected by this change, and to identify whether these same pathways are conversely affected with asthma. Continued research to identify other mechanisms leading to the altered risk of hypertensive disorders with smoking and asthma in pregnancy may assist in improving our understanding of the clinical course of this disease.

The national prevalence of asthma between 1999 and 2008 varied between 11\% and 14\% [3], indicating an under-reporting of asthma in this current cohort, where only $6.7 \%$ of pregnancies were associated with asthma. In the current study, the identification of asthma as a pregnancy complication was reliant upon it being recorded in the maternal antenatal or neonatal health records. This lower than expected prevalence of asthma potentially represents only those cases where an asthma-related event, such as an exacerbation, occurred during pregnancy. This figure would be consistent with previous prospective reports that identify exacerbations in $\sim 50 \%$ of asthmatic females during pregnancy [14, 42, 43]. Pregnant females who experience an asthma exacerbation requiring an emergency department visit, hospital admission and/or short course of oral corticosteroid therapy appear to be most at risk of SGA compared to pregnancies where no exacerbation occurs $[24,44,45]$. A limitation of the current study was the lack of data detailing disease severity or exacerbations, which would have enabled discrimination of these factors. Nonetheless, a previous retrospective cohort analysis conducted in an Australian population with comparable smoking rates, and where asthma was identified in $12 \%$ of pregnancies, demonstrated similar increases in perinatal complications with asthma, including SGA deliveries, urinary tract infections and hypertensive disorders [6]. Together, these data indicate that the adverse perinatal outcomes observed in the current cohort are not specific to the most severe cases (including asthma exacerbations) of asthma. 
Despite evidence indicating that females carrying a female fetus experience a deterioration of asthma symptoms, as well as an increased requirement for medication and increased circulating monocytes in pregnancy [28, 46-49], most previous studies failed to consider the sex of the infant in their analyses. In the current study, a sex-specific association was demonstrated, with the risk of SGA increased in male but not female neonates in pregnancies of females with asthma. In the few studies that have assessed neonatal outcomes according to fetal sex, asthma increased the risk of male neonates delivering SGA [6, 15], preterm and stillborn [6]. Similar outcomes for male neonates were also reported in a prospective study of asthma exacerbations during pregnancy [14]. Reduced female fetal growth is reported in studies where asthma was not treated with inhaled corticosteroids during pregnancy $[28,50]$, due to changes in placental cortisol metabolism [51, 52]; however, this reduction does not reach the criteria for SGA or IUGR. These findings lend support to the suggestion that the females with asthma identified in the birth records in this study are those associated with an asthma exacerbation and/or severe asthma, which result in male but not female growth retardation in utero.

In the current study, pregnancy outcomes were assessed over a 10-year period; therefore, it is likely that the database contained more than one pregnancy for a portion of the females. In a prospective study of 34 consecutive pregnancies with asthma, SCHATz et al. [53] observed a high concordance rate between the progression of asthma in the first and second pregnancy. The effect size observed in this study may therefore be exaggerated, with the inclusion of more than one pregnancy for each female, particularly given that females with asthma more frequently had parity equal or greater than three, compared to the reference group. We were unable to link individual females within our database due to privacy and ethical issues, therefore we assessed the risk of adverse maternal and neonatal events in primiparous females alone and observed similar magnitudes in the odds ratios for most outcomes. We conclude that the observed increased risk of a poor maternal and neonatal outcome in pregnancies associated with maternal asthma is not amplified by the inclusion of subsequent pregnancies.

Details of asthma medications were not recorded in the birth records and we were therefore unable to determine whether the adverse outcomes were related to poor asthma control, compliance with medications and/or the prescribed asthma medications themselves. In early pregnancy, the use of bronchodilators or high doses of inhaled corticosteroids $\left(>1000 \mu \mathrm{g} \cdot \mathrm{day}^{-1}\right)$ for asthma treatment have been linked with an increased risk of congenital malformations [54, 55], an outcome observed in the current study in pregnancies associated with asthma. However, most medications appear safe to use during pregnancy, with no reports of increased risk of pregnancy complications reported with inhaled corticosteroid or $\beta$-agonist use $[13,56-60]$ and recent evidence demonstrating that inhaled corticosteroids do not cross the placenta to exert effects on the fetus [61]. Indeed, national guidelines for the treatment of asthma during pregnancy recommend the continued use of the same pharmacological treatments during pregnancy that control asthma in the nonpregnant state $[62,63]$.

The current study was limited by having no information regarding asthma exacerbations or medications used during pregnancy. Future large-scale epidemiological studies that detail asthma severity and medication use, dose and compliance would be beneficial in determining the impact of asthma on pregnancy outcome. For example, the increased risk of urinary tract infections with both cigarette smoking and asthma may be due to the use of inhaled or oral steroids for asthma treatment, resulting in immunosuppression and increased suceptibility to infection. These future studies should also aim to incorporate other potential factors that may contribute to poor perinatal outcomes that were unavailable in the current dataset, including timing of smoking cessation and use of nicotine replacement therapy, body mass index, and number/frequency of antenatal care, to elucidate more completely the best way to manage females with asthma during pregnancy. Nonetheless, improved antenatal management of asthma and targeted programmes to reduce smoking in these pregnancies could significantly improve maternal and neonatal perinatal outcomes in this at-risk population.

\section{Acknowledgements}

We would like to thank all the midwives, neonatal nurses and other hospital staff who notified to the SA Perinatal Database.

\section{References}

Masoli M, Fabian D, Holt S, et al. The global burden of asthma: executive summary of the GINA Dissemination Committee report. Allergy 2004; 59: 469-478.

2 Bousquet J, Bousquet PJ, Godard P, et al. The public health implications of asthma. Bull World Health Organ 2005; 83: 548-554.

3 Australian Centre for Asthma Monitoring. Asthma in Australia 2008. Canberra, Australian Institute for Health and Welfare, 2008. 
Wilson DH, Adams RJ, Tucker G, et al. Trends in asthma prevalence and population changes in South Australia, 1990-2003. Med J Aust 2006; 184: 226-229.

5 Ghosh S, Pahwa P, Rennie D, et al. Opposing trends in the prevalence of health professional-diagnosed asthma by sex: a Canadian National Population Health Survey study. Can Respir J 2008; 15: 146-152.

6 Clifton VL, Engel P, Smith R, et al. Maternal and neonatal outcomes of pregnancies complicated by asthma in an Australian population. Aust NZ J Obstet Gynaecol 2009; 49: 619-626.

7 Demissie K, Breckenridge MB, Rhoads GG. Infant and maternal outcomes in the pregnancies of asthmatic women. Am J Respir Crit Care Med 1998; 158: 1091-1095.

8 Källén B, Rydhstroem H, Aberg A. Asthma during pregnancy - a population based study. Eur J Epidemiol 2000; 16: $167-171$.

9 Liu S, Wen SW, Demissie K, et al. Maternal asthma and pregnancy outcomes: a retrospective cohort study. Am J Obstet Gynecol 2001; 184: 90-96.

10 Blais L, Kettani FZ, Elftouh N, et al. Effect of maternal asthma on the risk of specific congenital malformations: a population-based cohort study. Birth Defects Res A Clin Mol Teratol 2010; 88: 216-222.

11 Murphy VE, Namazy JA, Powell H, et al. A meta-analysis of adverse perinatal outcomes in women with asthma. BJOG 2011; 118: 1314-1323.

12 Firoozi F, Lemière C, Ducharme FM, et al. Effect of maternal moderate to severe asthma on perinatal outcomes. Respir Med 2010; 104: 1278-1287.

13 Bakhireva LN, Schatz M, Jones KL, et al. Asthma control during pregnancy and the risk of preterm delivery or impaired fetal growth. Ann Allergy Asthma Immunol 2008; 101: 137-143.

14 Murphy VE, Gibson P, Talbot P, et al. Severe asthma exacerbations during pregnancy. Obstet Gynecol 2005; 106: 1046-1054.

15 Clark JM, Hulme E, Devendrakumar V, et al. Effect of maternal asthma on birthweight and neonatal outcome in a British inner-city population. Paediatr Perinat Epidemiol 2007; 21: 154-162.

16 Minerbi-Codish I, Fraser D, Avnun L, et al. Influence of asthma in pregnancy on labor and the newborn. Respiration 1998; 65: 130-135.

17 Stenius-Aarniala B, Piirilä P, Teramo K. Asthma and pregnancy: a prospective study of 198 pregnancies. Thorax 1988; 43: 12-18.

18 Firoozi F, Ducharme FM, Lemière C, et al. Effect of fetal gender on maternal asthma exacerbations in pregnant asthmatic women. Respir Med 2009; 103: 144-151.

19 Cnattingius S. The epidemiology of smoking during pregnancy: smoking prevalence, maternal characteristics, and pregnancy outcomes. Nicotine Tob Res 2004; 6: Suppl. 2, S125-S140.

20 George L, Granath F, Johansson AL, et al. Environmental tobacco smoke and risk of spontaneous abortion. Epidemiology 2006; 17: 500-505.

21 Räisänen S, Gissler M, Saari J, et al. Contribution of risk factors to extremely, very and moderately preterm births register-based analysis of 1,390,742 singleton births. PLoS One 2013; 8: e60660.

22 Ward C, Lewis S, Coleman T. Prevalence of maternal smoking and environmental tobacco smoke exposure during pregnancy and impact on birth weight: retrospective study using Millennium Cohort. BMC Public Health 2007; 7: 81.

23 DiFranza JR, Aligne CA, Weitzman M. Prenatal and postnatal environmental tobacco smoke exposure and children's health. Pediatrics 2004; 113: 1007-1015.

24 Enriquez R, Griffin MR, Carroll KN, et al. Effect of maternal asthma and asthma control on pregnancy and perinatal outcomes. J Allergy Clin Immunol 2007; 120: 625-630.

25 Kyrklund-Blomberg NB, Cnattingius S. Preterm birth and maternal smoking: risks related to gestational age and onset of delivery. Am J Obstet Gynecol 1998; 179: 1051-1055.

26 McLean A, Scott J, Keane R. Validation of the 1994 South Australian Perinatal Data Collection Form. Adelaide, Dept of Human Services, 2001.

27 Census of Population and Housing: Socio-Economic Indexes for Areas (SEIFA), Australia 2006. Report No.: 2033.0.55.001. Canberra, Australian Bureau of Statistics, 2008.

28 Murphy VE, Gibson PG, Giles WB, et al. Maternal asthma is associated with reduced female fetal growth. Am J Respir Crit Care Med 2003; 168: 1317-1323.

29 Andres RL, Day MC. Perinatal complications associated with maternal tobacco use. Semin Neonatol 2000; 5: $231-241$.

30 Henderson AJ, Sherriff A, Northstone K, et al. Pre- and postnatal parental smoking and wheeze in infancy: cross cultural differences. Eur Respir J 2001; 18: 323-329.

31 Le Souëf PN. Adverse effects of maternal smoking during pregnancy on innate immunity in infants. Eur Respir J 2006; 28: 675-677.

32 Clifford A, Lang L, Chen R. Effects of maternal cigarette smoking during pregnancy on cognitive parameters of children and young adults: a literature review. Neurotoxicol Teratol 2012; 34: 560-570.

33 Bruin JE, Gerstein HC, Holloway AC. Long-term consequences of fetal and neonatal nicotine exposure: a critical review. Toxicol Sci 2010; 116: 364-374.

34 Bahna SL, Bjerkedal T. The course and outcome of pregnancy in women with bronchial asthma. Acta Allergol 1972; 27: 397-406.

35 Shipton D, Tappin DM, Vadiveloo T, et al. Reliability of self reported smoking status by pregnant women for estimating smoking prevalence: a retrospective, cross sectional study. BMJ 2009; 339: b4347.

36 Tobacco Smoking in Australia: a Snapshot, 2004-05. Report No. 4831.0.55.001.Canberra, Australian Bureau of Statistics, 2006.

37 Mendola P, Laughon SK, Männistö TI, et al. Obstetric complications among US women with asthma. Am J Obstet Gynecol 2013; 208: 127.

Pietinalho A, Pelkonen A, Rytilä P. Linkage between smoking and asthma. Allergy 2009; 64: 1722-1727.

Thomson NC, Chaudhuri R, Livingston E. Asthma and cigarette smoking. Eur Respir J 2004; 24 : $822-833$.

England L, Zhang J. Smoking and risk of preeclampsia: a systematic review. Front Biosci 2007; 12: $2471-2483$.

Jeyabalan A, Powers RW, Durica AR, et al. Cigarette smoke exposure and angiogenic factors in pregnancy and preeclampsia. Am J Hypertens 2008; 21: 943-947.

42 Dombrowski MP, Brown CL, Berry SM. Preliminary experience with triamcinolone acetonide during pregnancy. J Matern Fetal Med 1996; 5: 310-313. 
43 Perlow JH, Montgomery D, Morgan MA, et al. Severity of asthma and perinatal outcome. Am J Obstet Gynecol 1992; 167: 963-967.

44 Greenberger PA, Patterson R. The outcome of pregnancy complicated by severe asthma. Allergy Proc 1988; 9: 539-543.

45 Fitzsimons R, Greenberger PA, Patterson R. Outcome of pregnancy in women requiring corticosteroids for severe asthma. J Allergy Clin Immunol 1986; 78: 349-353.

46 Beecroft N, Cochrane GM, Milburn HJ. Effect of sex of fetus on asthma during pregnancy: blind prospective study. BMJ 1998; 317: 856-857.

47 Dodds L, Armson BA, Alexander S. Use of asthma drugs is less among women pregnant with boys rather than girls. BMJ 1999; 318: 1011.

48 Kwon HL, Belanger K, Holford TR, et al. Effect of fetal sex on airway lability in pregnant women with asthma. Am J Epidemiol 2006; 163: 217-221.

49 Clifton VL, Murphy VE. Maternal asthma as a model for examining fetal sex-specific effects on maternal physiology and placental mechanisms that regulate human fetal growth. Placenta 2004; 25: Suppl. A, S45-S52.

50 Clifton VL. Sexually dimorphic effects of maternal asthma during pregnancy on placental glucocorticoid metabolism and fetal growth. Cell Tissue Res 2005; 322: 63-71.

51 Murphy VE, Zakar T, Smith R. Reduced 11ß-hydroxysteroid dehydrogenase type 2 activity is associated with decreased birth weight centile in pregnancies complicated by asthma. J Clin Endocrinol Metab 2002; 87: 1660-1668.

52 Hodyl NA, Wyper H, Osei-Kumah A, et al. Sex-specific associations between cortisol and birth weight in pregnancies complicated by asthma are not due to differential glucocorticoid receptor expression. Thorax 2010; 65: 677-683.

53 Schatz M, Harden K, Forsythe A, et al. The course of asthma during pregnancy, post partum, and with successive pregnancies: a prospective analysis. J Allergy Clin Immunol 1988; 81: 509-517.

54 Blais L, Beauchesne MF, Lemière C, et al. High doses of inhaled corticosteroids during the first trimester of pregnancy and congenital malformations. J Allergy Clin Immunol 2009; 124: 1229-1234.

55 Munsie JW, Lin S, Browne ML, et al. Maternal bronchodilator use and the risk of orofacial clefts. Hum Reprod 2011; 26: $3147-3154$

56 Schatz M, Dombrowski MP, Wise R, et al. The relationship of asthma medication use to perinatal outcomes. J Allergy Clin Immunol 2004; 113: 1040-1045.

57 Schatz M, Zeiger RS, Harden K, et al. The safety of asthma and allergy medications during pregnancy. J Allergy Clin Immunol 1997; 100: 301-306.

58 Martel MJ, Rey E, Beauchesne MF, et al. Use of inhaled corticosteroids during pregnancy and risk of pregnancy induced hypertension: nested case-control study. BMJ 2005; 330: 230.

59 Källén B, Rydhstroem H, Aberg A. Congenital malformations after the use of inhaled budesonide in early pregnancy. Obstet Gynecol 1999; 93: 392-395.

60 Namazy J, Schatz M, Long L, et al. Use of inhaled steroids by pregnant asthmatic women does not reduce intrauterine growth. J Allergy Clin Immunol 2004; 113: 427-432.

61 Hodyl NA, Stark MJ, Osei-Kumah A, et al. Fetal glucocorticoid-regulated pathways are not affected by inhaled corticosteroid use for asthma during pregnancy. Am J Respir Crit Care Med 2011; 183: 716-722.

62 National Asthma Council. Asthma Management Handbook. Melbourne, National Asthma Council Australia Ltd, 2006.

63 Busse WW. NAEPP Expert panel report. Managing asthma during pregnancy: recommendations for pharmacologic treatment - 2004 update. J Allergy Clin Immunol 2005; 115: 34-46. 